\title{
SOME ERROR ESTIMATES FOR THE LUMPED MASS FINITE ELEMENT METHOD FOR A PARABOLIC PROBLEM
}

\author{
P. CHATZIPANTELIDIS, R. D. LAZAROV, AND V. THOMÉE
}

\begin{abstract}
We study the spatially semidiscrete lumped mass method for the model homogeneous heat equation with homogeneous Dirichlet boundary conditions. Improving earlier results we show that known optimal order smooth initial data error estimates for the standard Galerkin method carry over to the lumped mass method whereas nonsmooth initial data estimates require special assumptions on the triangulation. We also discuss the application to time discretization by the backward Euler and Crank-Nicolson methods.
\end{abstract}

\section{INTRODUCTION}

We consider the model initial-boundary value problem

$$
\begin{aligned}
u_{t}-\Delta u & =0, \quad \text { in } \Omega, \quad u=0, \quad \text { on } \partial \Omega, \quad \text { for } t \geq 0, \\
u(0) & =v, \quad \text { in } \Omega,
\end{aligned}
$$

where $\Omega$ is a bounded convex polygonal domain in $\mathbb{R}^{2}$. For simplicity we restrict ourselves to the homogeneous heat equation, thus without a forcing term, so that the initial values $v$ are the only data of the problem. This problem has a unique solution $u(t)$, under appropriate assumptions on $v$, and this solution is smooth for $t>0$, even if $v$ is not. More precisely, for $q \geq 0$ we denote by $\dot{H}^{q} \subset L_{2}(\Omega)$ the Hilbert space defined by the norm

$$
|v|_{q}=\left(\sum_{j=1}^{\infty} \lambda_{j}^{q}\left(v, \phi_{j}\right)^{2}\right)^{1 / 2}, \quad \text { where }(v, w)=\int_{\Omega} v w d x,
$$

and where $\left\{\lambda_{j}\right\}_{j=1}^{\infty},\left\{\phi_{j}\right\}_{j=1}^{\infty}$ are the eigenvalues and eigenfunctions of $-\Delta$, with homogeneous Dirichlet boundary conditions on $\partial \Omega$. Thus $|v|_{0}=\|v\|=(v, v)^{1 / 2}$ is the norm in $L_{2}=L_{2}(\Omega),|v|_{1}$ the norm in $H_{0}^{1}=H_{0}^{1}(\Omega)$ and $|v|_{2}=\|\Delta v\|$ is equivalent to the norm in $H^{2}(\Omega)$ when $v=0$ on $\partial \Omega$. For the solution of (1.1) we then have the stability and smoothing estimate

$$
|E(t) v|_{p} \leq C t^{-(p-q) / 2}|v|_{q}, \quad \text { for } 0 \leq q \leq p, \quad t>0, \quad \text { where } u(t)=E(t) v .
$$

We first recall some facts about the spatially semidiscrete standard Galerkin method for (1.1) in the piecewise linear finite element space

$$
S_{h}=\left\{\chi \in \mathcal{C}(\Omega):\left.\chi\right|_{\tau} \text { linear, } \forall \tau \in \mathcal{T}_{h} ;\left.\chi\right|_{\partial \Omega}=0\right\},
$$

Received by the editor November 5, 2009 and, in revised form, November 2, 2010.

2010 Mathematics Subject Classification. Primary 65M60, 65M15.

Key words and phrases. Lumped mass method, parabolic partial differential equations, nonsmooth initial data, error estimates. 
where $\left\{\mathcal{T}_{h}\right\}_{0<h<1}$ is a family of regular triangulations $\mathcal{T}_{h}=\{\tau\}$ of $\Omega$, with $h$ denoting the maximum diameter of the triangles $\tau \in \mathcal{T}_{h}$. We then seek an approximation $u_{h}(t) \in S_{h}$ of $u(t)$ for $t \geq 0$ from

$$
\left(u_{h, t}, \chi\right)+\left(\nabla u_{h}, \nabla \chi\right)=0, \quad \forall \chi \in S_{h}, \quad \text { for } t \geq 0, \quad \text { with } u_{h}(0)=v_{h},
$$

where $v_{h} \in S_{h}$ is an approximation of $v$. It is well known that we have the smooth data error estimate, valid uniformly down to $t=0$; cf. [7, Theorem 3.1],

$$
\left\|u_{h}(t)-u(t)\right\| \leq C h^{2}|v|_{2}, \quad \text { for } t \geq 0, \quad \text { if } \quad\left\|v_{h}-v\right\| \leq C h^{2}|v|_{2} .
$$

We also have a nonsmooth data error estimate, for $v$ only assumed to be in $L_{2}$, but which deteriorates for $t$ tending to zero (cf. [7, Theorem 3.2]), namely

$$
\left\|u_{h}(t)-u(t)\right\| \leq C h^{2} t^{-1}\|v\|, \quad \text { if } \quad v_{h}=P_{h} v, \quad \text { for } t>0,
$$

where $P_{h}$ denotes the $L_{2}$-projection onto $S_{h}$. Note that the discrete initial data are not as general in this case as in (1.3).

We remark that the nonsmooth data error estimate (1.4) is of optimal order $O\left(h^{2}\right)$ for $t$ bounded away from zero, but deteriorates as $t \rightarrow 0$. We emphasize that the triangulations $\mathcal{T}_{h}$ are assumed to be independent of $t$, and thus that the use of finer $\mathcal{T}_{h}$ for $t$ small is not considered here.

We note for later use that a possible choice in (1.3) is $v_{h}=P_{h} v$, and that hence, by interpolation, we have the intermediate result between (1.3) and (1.4),

$$
\left\|u_{h}(t)-u(t)\right\| \leq C h^{2} t^{-1 / 2}|v|_{1}, \quad \text { if } v_{h}=P_{h} v, \quad \text { for } t>0 .
$$

As is easily seen, this error bound also holds for $v_{h}=R_{h} v$, the Ritz projection of $v$ onto $S_{h}$ defined in (2.4) below. In the sequel we shall not insist on generality in the choice of $v_{h}$ in our various error estimates, and an estimate such as (1.3) would be expressed with $v_{h}=R_{h} v$. The above more general choice of $v_{h}$ is then justified by the stability of (1.2) in $v_{h}$.

The object of study in this paper is the lumped mass modification of (1.2) obtained by replacing the first term on the left by a quadrature expression, or

$$
\left(\bar{u}_{h, t}, \chi\right)_{h}+\left(\nabla \bar{u}_{h}, \nabla \chi\right)=0, \quad \forall \chi \in S_{h}, \quad \text { for } t \geq 0, \quad \text { with } \bar{u}_{h}(0)=v_{h},
$$

where, denoting by $\left\{z_{j}^{\tau}\right\}_{j=1}^{3}$ the vertices of $\tau$ and by $\pi_{h}: \mathcal{C}(\bar{\Omega}) \rightarrow S_{h}$ the finite element interpolation operator,

$$
(v, w)_{h}=\sum_{\tau \in \mathcal{T}_{h}} Q_{\tau, h}(v w), \quad \text { with } Q_{\tau, h}(f)=\frac{|\tau|}{3} \sum_{j=1}^{3} f\left(z_{j}^{\tau}\right)=\int_{\tau} \pi_{h} f d x .
$$

This method has the advantage over the standard Galerkin method that, under the assumption that the triangulation is of Delaunay type, the solution satisfies the maximum-principle; cf., e.g. [7, Theorem 15.5]. Our aim here is to show analogues of (1.3), (1.4), and (1.5) for the solution of (1.6), namely, with the appropriate choices of $v_{h}$,

$$
\left\|\bar{u}_{h}(t)-u(t)\right\| \leq C h^{2} t^{-(1-q / 2)}|v|_{q}, \quad \text { for } t>0, \quad q=0,1,2 .
$$

We will prove this in Section 3 for $q=1,2$. However, for $q=0$, we are only able to show this under an additional hypothesis, expressed in terms of the quadrature error operator $Q_{h}: S_{h} \rightarrow S_{h}$, defined by

$$
\left(\nabla Q_{h} \chi, \nabla \psi\right)=\epsilon_{h}(\chi, \psi):=(\chi, \psi)_{h}-(\chi, \psi), \quad \forall \psi \in S_{h},
$$


and requiring that

$$
\left\|Q_{h} \psi\right\| \leq C h^{2}\|\psi\|, \quad \forall \psi \in S_{h} .
$$

It will be shown that this assumption is satisfied for symmetric triangulations. We will then give examples, in one space dimension, of nonsymmetric partitions such that (1.8) does not hold for $q=0$. For finite difference methods, which are close in character to the lumped mass method with symmetric triangulations, it was shown in [5], that nonsmooth data estimates similar to (1.8) with $q=0$ hold. Without condition (1.10) we are only able to show the nonoptimal order error estimate

$$
\left\|\bar{u}_{h}(t)-u(t)\right\| \leq C h t^{-1 / 2}\|v\|, \quad \text { for } t>0 .
$$

Symmetry of the triangulations is a serious restriction which can only hold for special shapes of $\Omega$.

We also discuss optimal order $O(h)$ error estimates for the gradient of $\bar{u}_{h}(t)-u(t)$, with a dependence of $t$ depending on the smoothness of $v$.

Our analysis provides improvements of earlier results in [3] (cf. also 7, Lemma 15.3 and p. 267]) where, by mimicking the proof for the standard Galerkin method, it was shown that, e.g.,

$$
\left\|\bar{u}_{h}(t)-u(t)\right\| \leq\left\{\begin{array}{l}
C h^{2}|v|_{3}, \\
C h^{2} t^{-1 / 2}|v|_{2},
\end{array} \quad \text { for } t>0, \quad \text { if } v_{h}=R_{h} v,\right.
$$

thus requiring more regularity of the initial data than (1.8). Our approach here is to combine the error estimates (1.3), (1.4) and (1.5) for the standard Galerkin method with new bounds for the difference $\delta(t)=\bar{u}_{h}(t)-u_{h}(t)$, which satisfies

$$
\left(\delta_{t}, \chi\right)_{h}+(\nabla \delta, \nabla \chi)=-\epsilon_{h}\left(u_{h, t}, \chi\right), \quad \text { for } \chi \in S_{h} .
$$

After we had finished our research, we became aware of the paper [6], where the smooth data error estimate (1.8), with $q=2$, is shown for a slightly more general parabolic equation and by a somewhat more lengthy argument than here. The nonsmooth data error estimate, with $q=0$, is also stated but with an incomplete proof.

The following is an outline of the paper. In Section 2, we introduce notation and give some preliminary material needed for the analysis of the lumped mass method. Further, we derive smooth and nonsmooth initial data estimates for the gradient of the error in the standard Galerkin method, which will be used in the sequel. In Section 3 we derive error estimates for the lumped mass method for initial data with basic smoothness, or $v \in \dot{H}^{q}$ with $q=1$,2. In Section 4 we show the optimal order error bound for $v \in L_{2}$ under the assumption that (1.10) holds. In Section 5 we show that this assumption is valid for symmetric meshes, and in Section 6, in one space dimension, that the symmetry requirement can be somewhat relaxed. In Section 7 we give two nonsymmetric partitions in one space dimension for which optimal $L_{2}$-convergence for nonsmooth data does not hold. Finally, in Section 8 we consider briefly the application of our results for the spatially semidiscrete problem to the fully discrete backward Euler and Crank-Nicolson lumped mass methods.

\section{Preliminaries}

In this section we recall some basic known facts for the spatially semidiscrete standard Galerkin and lumped mass methods. We introduce notation and show 
smoothing properties of the solution operators for these two semidiscrete methods and some other preliminary results needed in the sequel.

Introducing the discrete Laplacian $\Delta_{h}: S_{h} \rightarrow S_{h}$ by

$$
-\left(\Delta_{h} \psi, \chi\right)=(\nabla \psi, \nabla \chi), \quad \forall \psi, \chi \in S_{h},
$$

we may write the spatially discrete problem (1.2) as

$$
u_{h, t}-\Delta_{h} u_{h}=0, \quad \text { for } t \geq 0, \quad \text { with } u_{h}(0)=v_{h} \text {. }
$$

With $u_{h}(t)$ its solution we define the solution operator $E_{h}(t)=e^{\Delta_{h} t}$ of (2.1) by $u_{h}(t)=E_{h}(t) v_{h}$. Letting $\left\{\lambda_{j}^{h}\right\}_{j=1}^{N},\left\{\phi_{j}^{h}\right\}_{j=1}^{N}$ denote the eigenvalues and eigenfunctions of $-\Delta_{h}$, we have, by eigenfunction expansion,

$$
u_{h}(t)=E_{h}(t) v_{h}=\sum_{j=1}^{N} e^{-\lambda_{j}^{h} t}\left(v_{h}, \phi_{j}^{h}\right) \phi_{j}^{h}, \quad \text { for } t \geq 0 .
$$

We shall need various smoothing properties of $E_{h}(t)$. First, we recall the following smoothing bounds for the exact solution $u$ of (1.1); cf., e.g., [7],

$$
\left|D_{t}^{\ell} E(t) v\right|_{p} \leq C t^{-\ell-(p-q) / 2}|v|_{q}, \quad \text { for } t>0, p, q, \ell \geq 0,2 \ell+p \geq q, v \in \dot{H}^{q} .
$$

In the following lemma, we show some discrete analogues of these bounds.

Lemma 2.1. For $E_{h}(t)$ defined by (2.2) we have, for $v_{h} \in S_{h}$,

$\left\|\nabla^{p} D_{t}^{\ell} E_{h}(t) v_{h}\right\| \leq C t^{-\ell-(p-q) / 2}\left\|\nabla^{q} v_{h}\right\|, \quad$ for $t>0, \ell \geq 0, p, q=0,1,2 \ell+p \geq q$.

Proof. By Parseval's relation, since $\lambda^{s} e^{-\lambda t} \leq C_{s} t^{-s}$, for $\lambda>0, s>0$, we get

$$
\left\|\nabla^{p} D_{t}^{\ell} E_{h}(t) v_{h}\right\|^{2}=\sum_{j=1}^{N}\left(\lambda_{j}^{h}\right)^{2 \ell+p} e^{-2 \lambda_{j}^{h} t}\left(v_{h}, \phi_{j}^{h}\right)^{2} \leq C t^{-2 \ell-p+q}\left\|\nabla^{q} v_{h}\right\|^{2} .
$$

In addition to the $L_{2}$-projection $P_{h}: L_{2} \rightarrow S_{h}$, satisfying

$$
\left(P_{h} v, \chi\right)=(v, \chi), \quad \forall \chi \in S_{h},
$$

our error analysis will use the Ritz projection, $R_{h}: H_{0}^{1} \rightarrow S_{h}$, defined by

$$
\left(\nabla R_{h} v, \nabla \chi\right)=(\nabla v, \nabla \chi), \quad \text { for } \chi \in S_{h}
$$

It is well known, (cf. e.g. [7, Lemma 1.1]) that $R_{h}$ satisfies

$$
\left\|R_{h} v-v\right\|+h\left\|\nabla\left(R_{h} v-v\right)\right\| \leq C h^{q}|v|_{q}, \quad \text { for } v \in \dot{H}^{q}, q=1,2 .
$$

Next, we turn to the lumped mass method. As is well known, the norms $\|\cdot\|_{h}$ and $\|\cdot\|$ are equivalent on $S_{h}$, or, more precisely,

$$
\frac{1}{2}\|\chi\|_{h} \leq\|\chi\| \leq\|\chi\|_{h}, \quad \forall \chi \in S_{h} .
$$

We now introduce the discrete Laplacian $-\bar{\Delta}_{h}: S_{h} \rightarrow S_{h}$, corresponding to the inner product $(\cdot, \cdot)_{h}$, by

$$
-\left(\bar{\Delta}_{h} \psi, \chi\right)_{h}=(\nabla \psi, \nabla \chi), \quad \forall \psi, \chi \in S_{h} .
$$

The lumped mass method (1.6) can then be written in operator form as

$$
\bar{u}_{h, t}-\bar{\Delta}_{h} \bar{u}_{h}=0, \quad \text { for } t \geq 0, \quad \text { with } \bar{u}_{h}(0)=v_{h} \text {. }
$$

With $\bar{E}_{h}(t)=e^{\bar{\Delta}_{h} t}$ as the solution operator of (2.8), we have

$$
\bar{u}_{h}(t)=\bar{E}_{h}(t) v_{h}=\sum_{j=1}^{N} e^{-\bar{\lambda}_{j}^{h} t}\left(v_{h}, \bar{\phi}_{j}^{h}\right)_{h} \bar{\phi}_{j}^{h},
$$


where $\left\{\bar{\lambda}_{j}^{h}\right\}_{j=1}^{N}$ and $\left\{\bar{\phi}_{j}^{h}\right\}_{j=1}^{N}$ are the eigenvalues and the corresponding orthonormal eigenfunctions, with respect to $(\cdot, \cdot)_{h}$, of the positive definite operator $-\bar{\Delta}_{h}$. We show the following analogue of Lemma 2.1 for $\bar{E}_{h}(t)$.

Lemma 2.2. For $\bar{E}_{h}(t)$ defined by (2.9) we have, for $v_{h} \in S_{h}$, $\left\|\nabla^{p} D_{t}^{\ell} \bar{E}_{h}(t) v_{h}\right\| \leq C t^{-\ell-(p-q) / 2}\left\|\nabla^{q} v_{h}\right\|, \quad$ for $t>0, \ell \geq 0, p, q=0,1,2 \ell+p \geq q$.

Proof. Introducing the square root $\bar{G}_{h}=\left(-\bar{\Delta}_{h}\right)^{1 / 2}: S_{h} \rightarrow S_{h}$, of $-\bar{\Delta}_{h}$, we have

$$
\left\|\nabla v_{h}\right\|^{2}=\left(\left(-\bar{\Delta}_{h}\right) v_{h}, v_{h}\right)_{h}=\left\|\bar{G}_{h} v_{h}\right\|_{h}^{2}=\sum_{j=1}^{N} \bar{\lambda}_{j}^{h}\left(v_{h}, \bar{\phi}_{j}^{h}\right)_{h}^{2} .
$$

Since the norms $\|\cdot\|_{h}$ and $\|\cdot\|$ are equivalent on $S_{h}$ we find

$$
\begin{aligned}
\left\|\nabla^{p} D_{t}^{\ell} \bar{E}_{h}(t) v_{h}\right\|^{2} & \leq C\left\|\bar{G}_{h}^{p} D_{t}^{\ell} \bar{E}_{h}(t) v_{h}\right\|_{h}^{2}=C \sum_{j=1}^{N}\left(\bar{\lambda}_{j}^{h}\right)^{2 \ell+p-q} e^{-2 \bar{\lambda}_{j}^{h} t}\left(\bar{\lambda}_{j}^{h}\right)^{q}\left(v_{h}, \bar{\phi}_{j}^{h}\right)_{h}^{2} \\
& \leq C t^{-(2 \ell+p-q)}\left\|\bar{G}_{h}^{q} v_{h}\right\|_{h}^{2} \leq C t^{-(2 \ell+p-q)}\left\|\nabla^{q} v_{h}\right\|^{2}
\end{aligned}
$$

We recall the following estimate for the error in the quadrature expression in (1.7).

Lemma 2.3. Let $\epsilon_{h}(\chi, \psi)=(\chi, \psi)_{h}-(\chi, \psi)$. Then

$$
\left|\epsilon_{h}(\chi, \psi)\right| \leq C h^{p+q}\left\|\nabla^{p} \chi\right\|\left\|\nabla^{q} \psi\right\|, \quad \forall \chi, \psi \in S_{h}, \quad \text { with } p, q=0,1 .
$$

Proof. For completeness we sketch the proof; cf. Lemma 15.1 in [7]. Since the quadrature formula is exact for linear functions over any triangle $\tau \in \mathcal{T}_{h}$, employing the Bramble-Hilbert lemma and a Sobolev inequality, we conclude that

$$
\left|Q_{\tau, h}(\chi \psi)-\int_{\tau} \chi \psi d x\right| \leq C h_{\tau}^{2} \sum_{|\alpha|=2}\left\|D^{\alpha}(\chi \psi)\right\|_{L_{1}(\tau)} \leq C h_{\tau}^{2}\|\nabla \chi\|_{L_{2}(\tau)}\|\nabla \psi\|_{L_{2}(\tau)},
$$

with $h_{\tau}$ the maximal side length of $\tau$. Now using an inverse inequality locally and summing over $\tau \in \mathcal{T}_{h}$, we obtain the desired result.

The following estimate holds for the quadrature error operator $Q_{h}$.

Lemma 2.4. Let $\bar{\Delta}_{h}$ and $Q_{h}$ be the operators defined by (2.7) and (1.9), respectively. Then

$$
\left\|\nabla Q_{h} \chi\right\|+h\left\|\bar{\Delta}_{h} Q_{h} \chi\right\|_{h} \leq C h^{p+1}\left\|\nabla^{p} \chi\right\|, \quad \forall \chi \in S_{h}, p=0,1 .
$$

Proof. By (1.9) and Lemma 2.3, with $\psi=Q_{h} \chi$ and $q=1$, it follows easily that

$$
\left\|\nabla Q_{h} \chi\right\|^{2}=\epsilon_{h}\left(\chi, Q_{h} \chi\right) \leq C h^{p+1}\left\|\nabla^{p} \chi\right\|\left\|\nabla Q_{h} \chi\right\|, \quad \text { for } p=0,1,
$$

which shows the first estimate of (2.10). Also, by the definition of $\bar{\Delta}_{h}$, Lemma 2.3 with $q=0$ shows

$$
\left\|\bar{\Delta}_{h} Q_{h} \chi\right\|_{h}^{2}=-\left(\nabla Q_{h} \chi, \nabla \bar{\Delta}_{h} Q_{h} \chi\right)=-\epsilon_{h}\left(\chi, \bar{\Delta}_{h} Q_{h} \chi\right) \leq C h^{p}\left\|\nabla^{p} \chi\right\|\left\|\bar{\Delta}_{h} Q_{h} \chi\right\|_{h},
$$

for $p=0,1$, which gives the second bound in (2.10).

In our analysis of the lumped mass method (1.6) we shall be interested not only in the error estimates (1.8) but also in the corresponding estimates for the gradient of the error. Our approach will then require the following estimates for the standard Galerkin method. 
Theorem 2.1. Let $u$ and $u_{h}$ be the solutions of (1.1) and (1.2), respectively. Then

$$
\left\|\nabla\left(u_{h}(t)-u(t)\right)\right\| \leq \begin{cases}C h t^{-(1-q / 2)}|v|_{q}, & \text { for } q=1,2, \\ C h t^{-1}\|v\|, & \text { if } v_{h}=R_{h} v, \\ & \text { if } v_{h}=P_{h} v .\end{cases}
$$

Proof. In a customary way we split the error into two terms as

$$
u_{h}-u=\left(u_{h}-R_{h} u\right)+\left(R_{h} u-u\right)=\vartheta+\varrho .
$$

By (2.5) and (2.3) we have

$$
\|\nabla \varrho(t)\| \leq C h|u(t)|_{2} \leq C h t^{-1+q / 2}|v|_{q}, \quad \text { for } t>0, \quad v \in \dot{H}^{q}, q=0,1,2 .
$$

It remains to bound $\nabla \vartheta$ analogously. By our definitions we have

$$
\vartheta_{t}-\Delta_{h} \vartheta=-P_{h} \varrho_{t}, \quad \text { for } t>0 .
$$

In the cases $q=1,2$ the Ritz projection $R_{h} v$ is well defined so that $\vartheta(0)=0$ and hence, by Duhamel's principle,

$$
\vartheta(t)=-\int_{0}^{t} E_{h}(t-s) P_{h} \varrho_{t}(s) d s
$$

Using Lemma 2.1. the stability of $P_{h}$, (2.5) and (2.3), we find, for $2 p+1 \geq q$,

$$
\begin{aligned}
& \left\|\nabla D_{t}^{\ell} E_{h}(t-s) P_{h} D_{t}^{p} \varrho(s)\right\| \leq C(t-s)^{-\ell-1 / 2}\left\|D_{t}^{p} \varrho(s)\right\| \\
& \quad \leq C h(t-s)^{-\ell-1 / 2}\left|D_{t}^{p} u(s)\right|_{1} \leq C h(t-s)^{-\ell-1 / 2} s^{-p-1 / 2+q / 2}|v|_{q} .
\end{aligned}
$$

When $q=2$ we use this in (2.12) to obtain

$$
\|\nabla \vartheta(t)\| \leq C h \int_{0}^{t}(t-s)^{-1 / 2} s^{-1 / 2} d s|v|_{2}=C h|v|_{2},
$$

which shows the desired estimate for $\nabla \vartheta$ in this case.

To treat the case $q=1$ we use (2.12) to write

$$
\nabla \vartheta(t)=-\left\{\int_{0}^{t / 2}+\int_{t / 2}^{t}\right\} \nabla E_{h}(t-s) P_{h} \varrho_{t}(s) d s=T_{1}+T_{2} .
$$

Using (2.13), we find

$$
\left\|T_{2}\right\| \leq C h \int_{t / 2}^{t}(t-s)^{-1 / 2} s^{-1} d s|v|_{1} \leq C h t^{-1 / 2}|v|_{1} .
$$

For $T_{1}$ we obtain by integration by parts

$$
T_{1}=-\left[\nabla E_{h}(t-s) P_{h} \varrho(s)\right]_{0}^{t / 2}+\int_{0}^{t / 2} \nabla D_{s} E_{h}(t-s) P_{h} \varrho(s) d s,
$$

and hence

$$
\left\|T_{1}\right\| \leq C h t^{-1 / 2}|v|_{1}+C h \int_{0}^{t / 2}(t-s)^{-3 / 2} d s|v|_{1} \leq C h t^{-1 / 2}|v|_{1} .
$$

Together these estimates show the desired bound for $\nabla \vartheta$ for $q=1$.

Finally, for $q=0$, we multiply (2.11) by $t$ to obtain

$$
(t \vartheta)_{t}-\Delta_{h}(t \vartheta)=-t P_{h} \varrho_{t}+\vartheta, \quad \text { for } t>0 .
$$

Although $\vartheta(0)=v_{h}-R_{h} v$ is not defined when $v \notin H_{0}^{1}$, we have $t \vartheta(t) \rightarrow 0$ as $t \rightarrow 0$. Indeed, using the estimate (cf. [7, formula (3.12)])

$$
\left\|u_{h}(t)-u(t)\right\| \leq C h t^{-1 / 2}\|v\|,
$$


the error bound (2.5), and the regularity estimate $|u(t)|_{1} \leq C t^{-1 / 2}\|v\|$ we get

$$
\|\vartheta(t)\| \leq\left\|u_{h}(t)-u(t)\right\|+\left\|R_{h} u(t)-u(t)\right\| \leq C h t^{-1 / 2}\|v\|,
$$

which shows that $t \vartheta(t) \rightarrow 0$ as $t \rightarrow 0$.

Hence we may integrate the above equation over $(0, t)$ to find

$$
t \vartheta(t)=-\int_{0}^{t} s E_{h}(t-s) P_{h} \varrho_{t}(s) d s+\int_{0}^{t} E_{h}(t-s) \vartheta(s) d s,
$$

so that

$$
t \nabla \vartheta(t)=-\int_{0}^{t} s \nabla E_{h}(t-s) P_{h} \varrho_{t}(s) d s+\int_{0}^{t} \nabla E_{h}(t-s) \vartheta(s) d s=T_{3}+T_{4} .
$$

Using (2.13) with $l=0, p=1, q=0$ we obtain

$$
\left\|T_{3}\right\| \leq C h \int_{0}^{t}(t-s)^{-1 / 2} s^{-1 / 2} d s\|v\|=C h\|v\| .
$$

For $T_{4}$, we note that in view of (2.15) we have

$$
\left\|T_{4}\right\| \leq C h \int_{0}^{t}(t-s)^{-1 / 2} s^{-1 / 2} d s\|v\|=C h\|v\| .
$$

Together these estimates complete the proof for $q=0$ and thus of the theorem. Note that the choice $v_{h}=P_{h} v$ enters in the estimate for $u_{h}(t)-u(t)$ in (2.15).

\section{The lumped mass method With SMOOTh initial Data}

In this section we derive optimal order error estimates for the lumped mass method (1.6), with initial data $v$ in $\dot{H}^{2}$ and $\dot{H}^{1}$.

Theorem 3.1. Let $u$ be the solution of (1.1) and $\bar{u}_{h}$ that of (1.6). Then

$\left\|\bar{u}_{h}(t)-u(t)\right\|+h\left\|\nabla\left(\bar{u}_{h}(t)-u(t)\right)\right\| \leq C h^{2} t^{-(1-q / 2)}|v|_{q}, \quad$ for $q=1,2$, if $v_{h}=R_{h} v$.

Proof. Since the corresponding error bounds hold for the solution $u_{h}$ of the standard Galerkin method, by (1.3), (1.5) and Theorem 2.1, it suffices to show that

$\|\delta(t)\|+h\|\nabla \delta(t)\| \leq C h^{2} t^{-(1-q / 2)}|v|_{q}, \quad$ for $t>0, q=1,2, \quad$ where $\delta=\bar{u}_{h}-u_{h}$.

By (1.6), (1.2) and the definition (1.9) of the quadrature error operator $Q_{h}, \delta(t)$ satisfies (1.11) and hence

$$
\delta_{t}-\bar{\Delta}_{h} \delta=\bar{\Delta}_{h} Q_{h} u_{h, t}, \quad \text { for } t \geq 0, \quad \text { with } \delta(0)=0 .
$$

By Duhamel's principle this shows

$$
\delta(t)=\int_{0}^{t} \bar{E}_{h}(t-s) \bar{\Delta}_{h} Q_{h} u_{h, t}(s) d s .
$$

Using the fact that $\bar{E}_{h}(t) \bar{\Delta}_{h}=D_{t} \bar{E}_{h}(t)$, and Lemmas 2.2 and 2.4 we easily get

$$
\begin{aligned}
& \left\|\bar{E}_{h}(t) \bar{\Delta}_{h} Q_{h} \chi\right\|+h\left\|\nabla \bar{E}_{h}(t) \bar{\Delta}_{h} Q_{h} \chi\right\| \\
& \leq C t^{-1 / 2}\left(\left\|\nabla Q_{h} \chi\right\|+h\left\|\bar{\Delta}_{h} Q_{h} \chi\right\|\right) \leq C h^{2} t^{-1 / 2}\|\nabla \chi\|,
\end{aligned}
$$

and hence

$$
\|\delta(t)\|+h\|\nabla \delta(t)\| \leq C h^{2} \int_{0}^{t}(t-s)^{-1 / 2}\left\|\nabla u_{h, t}(s)\right\| d s
$$


Here, since $\Delta_{h} R_{h}=P_{h} \Delta$, we obtain, by first applying Lemma 2.1 $\left\|\nabla u_{h, t}(s)\right\| \leq C s^{-1 / 2}\left\|u_{h, t}(0)\right\|=C s^{-1 / 2}\left\|\Delta_{h} R_{h} v\right\|=C s^{-1 / 2}\left\|P_{h} \Delta v\right\| \leq C s^{-1 / 2}|v|_{2}$, and hence

$$
\|\delta(t)\|+h\|\nabla \delta(t)\| \leq C h^{2} \int_{0}^{t}(t-s)^{-1 / 2} s^{-1 / 2} d s|v|_{2}=C h^{2}|v|_{2},
$$

which completes the proof for $q=2$.

To treat the case $q=1$, we use (3.2) to write

$$
\delta(t)=\left\{\int_{0}^{t / 2}+\int_{t / 2}^{t}\right\} \bar{E}_{h}(t-s) \bar{\Delta}_{h} Q_{h} u_{h, t}(s) d s=\delta_{1}(t)+\delta_{2}(t) .
$$

Here we have, in the same way as above,

$$
\begin{aligned}
&\left\|\delta_{2}(t)\right\|+ h\left\|\nabla \delta_{2}(t)\right\| \leq C h^{2} \int_{t / 2}^{t}(t-s)^{-1 / 2}\left\|\nabla u_{h, t}(s)\right\| d s \\
& \leq C h^{2} \int_{t / 2}^{t}(t-s)^{-1 / 2} s^{-1} d s\left\|\nabla R_{h} v\right\| \leq C h^{2} t^{-1 / 2}|v|_{1} .
\end{aligned}
$$

Integrating by parts we obtain

$$
\delta_{1}(t)=\left[\bar{E}_{h}(t-s) \bar{\Delta}_{h} Q_{h} u_{h}(s)\right]_{0}^{t / 2}-\int_{0}^{t / 2} D_{s} \bar{E}_{h}(t-s) \bar{\Delta}_{h} Q_{h} u_{h}(s) d s .
$$

Employing (3.3) we now find, similarly to the above,

$$
\begin{aligned}
\left\|\delta_{1}(t)\right\| & +h\left\|\nabla \delta_{1}(t)\right\| \leq C h^{2} t^{-1 / 2}\left(\left\|\nabla u_{h}(t / 2)\right\|+\left\|\nabla R_{h} v\right\|\right) \\
& +C h^{2} \int_{0}^{t / 2}(t-s)^{-3 / 2}\left\|\nabla u_{h}(s)\right\| d s \leq C h^{2} t^{-1 / 2}|v|_{1} .
\end{aligned}
$$

Together these estimates complete the proof.

\section{The Lumped MASS METHOD WITH NONSMOOTH INITIAL DATA}

In this section we discuss error estimates for the lumped mass method with $v \in L_{2}$, with discrete initial data $v_{h}=P_{h} v$. To derive an optimal order error bound analogous to (1.4) for the standard Galerkin method, we now need to impose a condition on the triangulations $\mathcal{T}_{h}$, expressed as a boundedness condition for the quadrature error operator $Q_{h}$. Without this condition we are only able to show a nonoptimal order $O(h)$ error bound in $L_{2}$, whereas for the gradient of the error an optimal order $O(h)$ still holds. We begin with the following theorem.

Theorem 4.1. Let $u$ be the solution of (1.1) with $v \in L_{2}$ and let $\bar{u}_{h}$ be the solution of (1.6), with $v_{h}=P_{h} v$. Then

$$
\left\|\bar{u}_{h}(t)-u(t)+\bar{E}_{h}(t) \bar{\Delta}_{h} Q_{h} v_{h}\right\| \leq C h^{2} t^{-1}\|v\|, \quad \text { for } t>0 .
$$

Hence, a necessary and sufficient condition for the nonsmooth data error bound

$$
\left\|\bar{u}_{h}(t)-u(t)\right\| \leq C h^{2} t^{-1}\|v\|, \quad \text { for } t>0,
$$

is that

$$
\left\|\bar{E}_{h}(t) \bar{\Delta}_{h} Q_{h} P_{h} v\right\| \leq C h^{2} t^{-1}\|v\|, \quad \text { for } t>0 .
$$


Proof. Using again the notation $\delta=\bar{u}_{h}-u_{h}$, we write

$$
\bar{u}_{h}(t)-u(t)+\bar{E}_{h}(t) \bar{\Delta}_{h} Q_{h} P_{h} v=\left(\delta(t)+\bar{E}_{h}(t) \bar{\Delta}_{h} Q_{h} P_{h} v\right)+\left(u_{h}(t)-u(t)\right) .
$$

Thus, in view of (1.4), it suffices to bound $\delta(t)+\bar{E}_{h}(t) \bar{\Delta}_{h} Q_{h} P_{h} v$. Using the representation (3.4) and (3.5) of $\delta$, we obtain

$$
\begin{aligned}
\delta(t)+\bar{E}_{h}(t) \bar{\Delta}_{h} Q_{h} P_{h} v= & \bar{E}_{h}(t / 2) \bar{\Delta}_{h} Q_{h} u_{h}(t / 2)+\delta_{2}(t)+\delta_{3}(t), \\
& \text { where } \delta_{3}(t)=-\int_{0}^{t / 2} D_{s} \bar{E}_{h}(t-s) \bar{\Delta}_{h} Q_{h} u_{h}(s) d s .
\end{aligned}
$$

Here, similarly to the proof of Theorem 3.1. using the stability of $P_{h}$,

$$
\left\|\bar{E}_{h}(t / 2) \bar{\Delta}_{h} Q_{h} u_{h}(t / 2)\right\| \leq C h^{2} t^{-1 / 2}\left\|\nabla u_{h}(t / 2)\right\| \leq C h^{2} t^{-1}\left\|P_{h} v\right\| \leq C h^{2} t^{-1}\|v\| .
$$

Further,

$$
\left\|\delta_{2}(t)\right\| \leq C h^{2} \int_{t / 2}^{t}(t-s)^{-1 / 2} s^{-3 / 2} d s\left\|P_{h} v\right\| \leq C h^{2} t^{-1}\|v\|,
$$

and, since $\left\|\nabla u_{h}(s)\right\| \leq C s^{-1 / 2}\left\|P_{h} v\right\|$,

$$
\left\|\delta_{3}(t)\right\| \leq C h^{2} \int_{0}^{t / 2}(t-s)^{-3 / 2}\left\|\nabla u_{h}(s)\right\| d s \leq C h^{2} t^{-1}\|v\| .
$$

Together these estimates show the desired bound (4.1).

We will now use this result to show that the $O\left(h^{2}\right)$ error bound (1.10) for the quadrature error operator $Q_{h}: S_{h} \rightarrow S_{h}$ defined in (1.9) is sufficient for the nonsmooth data error estimate (4.2) to hold.

Theorem 4.2. Let $u$ be the solution of (1.1) with $v \in L_{2}$ and let $\bar{u}_{h}$ be the solution of (1.6), with $v_{h}=P_{h} v$. Assume that $Q_{h}$ satisfies (1.10). Then (4.2) holds.

Proof. The result follows by Theorem 4.1 since, by Lemma 2.2 and (1.10), we have

$$
\left\|\bar{E}_{h}(t) \bar{\Delta}_{h} Q_{h} v_{h}\right\| \leq C t^{-1}\left\|Q_{h} v_{h}\right\| \leq C h^{2} t^{-1}\|v\| .
$$

The condition (1.10) will be discussed in more detail in Section 5 below. Note that, by Lemma 2.4, without additional assumptions on the mesh, we always have

$$
\left\|Q_{h} \chi\right\| \leq C\left\|\nabla Q_{h} \chi\right\| \leq C h\|\chi\|, \quad \forall \psi \in S_{h},
$$

and that the following lower order error estimate always holds.

Theorem 4.3. Let $u$ be the solution of (1.1) with $v \in L_{2}$ and $\bar{u}_{h}$ the solution of (1.6), with $v_{h}=P_{h} v$. Then

$$
\left\|\bar{u}_{h}(t)-u(t)\right\| \leq C h t^{-1 / 2}\|v\|, \quad \text { for } t>0 .
$$

Proof. Using the stability of $\bar{E}_{h}(t)$ and $E(t)$, and Lemma 2.4 we find

$$
\left\|\bar{u}_{h}(t)-u(t)+\bar{E}_{h}(t) \bar{\Delta}_{h} Q_{h} P_{h} v\right\| \leq C\|v\|+C\left\|\bar{\Delta}_{h} Q_{h} P_{h} v\right\| \leq C\|v\| .
$$

Combining this estimate with (4.1), we obtain

$$
\left\|\bar{u}_{h}(t)-u(t)+\bar{E}_{h}(t) \bar{\Delta}_{h} Q_{h} v_{h}\right\| \leq C h t^{-1 / 2}\|v\| .
$$

But by Lemmas 2.2 and 2.4, we have

$$
\left\|\bar{E}_{h}(t) \bar{\Delta}_{h} Q_{h} v_{h}\right\| \leq C t^{-1 / 2}\left\|\nabla Q_{h} P_{h} v\right\| \leq C h t^{-1 / 2}\|v\|,
$$

which shows the desired bound. 
We end this section by showing an optimal order $H^{1}$-norm nonsmooth data error estimate, which does not require the additional assumption (1.10).

Theorem 4.4. Let $u$ the solution of (1.1) with $v \in L_{2}$ and $\bar{u}_{h}$ the solution of (1.6), with $v_{h}=P_{h} v$. Then

$$
\left\|\nabla\left(\bar{u}_{h}(t)-u(t)\right)\right\| \leq C h t^{-1}\|v\|, \quad \text { for } t>0 .
$$

Proof. In view of Theorem 2.1 it suffices to show

$$
\|\nabla \delta(t)\| \leq C h t^{-1}\|v\|, \quad \text { for } t>0
$$

Multiplying (3.1) by $t$, we get

$$
(t \delta)_{t}-\bar{\Delta}_{h}(t \delta)=t \bar{\Delta}_{h} Q_{h} u_{h, t}+\delta, \quad \text { for } t \geq 0
$$

Hence, by Duhamel's principle we get

$$
t \nabla \delta(t)=\int_{0}^{t} s \nabla \bar{E}_{h}(t-s) \bar{\Delta}_{h} Q_{h} u_{h, t}(s) d s+\int_{0}^{t} \nabla \bar{E}_{h}(t-s) \delta(s) d s=I+I I .
$$

By (3.3), Lemma 2.1 and the stability of $P_{h}$, we find

$$
\|I\| \leq C h \int_{0}^{t}(t-s)^{-1 / 2} s^{-1 / 2} d s\left\|v_{h}\right\| \leq C h\|v\| .
$$

To bound $I I$, we use (2.14) and Theorem 4.3 to obtain

$$
\|\delta(t)\| \leq\left\|\bar{u}_{h}(t)-u(t)\right\|+\left\|u_{h}(t)-u(t)\right\| \leq C h t^{-1 / 2}\|v\| .
$$

Therefore, Lemma 2.2 gives

$$
\|I I\| \leq C h \int_{0}^{t}(t-s)^{-1 / 2} s^{-1 / 2} d s\|v\|=C h\|v\| .
$$

Together these estimates show (4.4), which completes the proof.

\section{Symmetric triangulations}

In this section we show that for triangulations that are symmetric, in a sense to be defined, assumption (1.10) is satisfied and therefore, by Theorem 4.2. the optimal order nonsmooth data error estimate (4.2) holds.
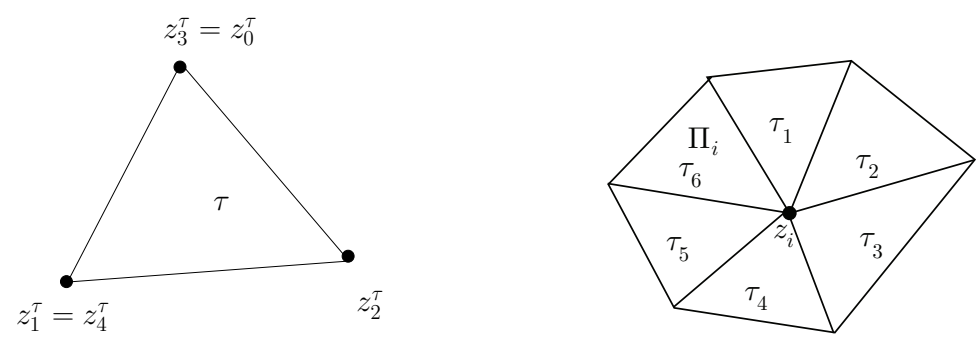

Figure 1. A triangle $\tau$ and a patch $\Pi_{i}$ around a vertex $z_{i}$

For $z_{i}$ a vertex of the triangulation $\mathcal{T}_{h}$ we define the patch $\Pi_{i}=\{\bigcup \tau: \tau \in$ $\left.\mathcal{T}_{h}, z_{i} \in \partial \tau\right\}$ (see Figure 11), and say that $\mathcal{T}_{h}$ is symmetric at $z_{i}$ if the patch $\Pi_{i}$ is symmetric around $z_{i}$, in the sense that if $x \in \Pi_{i}$, then $z_{i}-\left(x-z_{i}\right)=2 z_{i}-x \in \Pi_{i}$. Denoting by $Z_{h}^{0}$ the interior vertices of $\mathcal{T}_{h}$, we say that $\mathcal{T}_{h}$ is symmetric if it is symmetric at each $z_{i} \in Z_{h}^{0}$. The patch in Figure 1 is nonsymmetric with respect 
to $z_{i}$, whereas triangulations which are periodic repetition of the patches shown on Figure 2 are symmetric. Symmetric triangulations exist only for special domains, such as rectangles, but not for general polygonal domains.
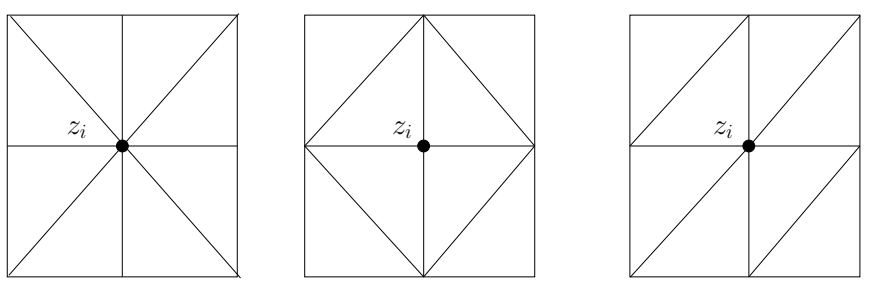

FIgURE 2. Patches which are symmetric with respect to the vertex $z_{i}$

We now show the sufficiency of symmetry for condition (1.10).

Theorem 5.1. If the triangulation $\mathcal{T}_{h}$ is symmetric, then (1.10) holds.

Proof. The proof is based on duality. For given $\chi \in S_{h}$ we define $\phi=\phi_{\chi} \in \dot{H}^{1}$ as the solution of the Dirichlet problem $(\nabla \phi, \nabla \eta)=(\chi, \eta)$ for all $\eta \in \dot{H}^{1}$. Since $\Omega$ is convex we have $\phi \in \dot{H}^{2}$ and $|\phi|_{2} \leq C\|\chi\|$. Letting $\pi_{h}$ be the finite element interpolation operator into $S_{h}$, we then have, for any $\psi \in S_{h}$,

$$
\begin{aligned}
\left\|Q_{h} \psi\right\| & =\sup _{\chi \in S_{h}} \frac{\left(Q_{h} \psi, \chi\right)}{\|\chi\|}=\sup _{\chi \in S_{h}} \frac{\left(\nabla Q_{h} \psi, \nabla \phi\right)}{\|\chi\|} \\
& \leq \sup _{\chi \in S_{h}} \frac{\left|\left(\nabla Q_{h} \psi, \nabla\left(\phi-\pi_{h} \phi\right)\right)\right|}{\|\chi\|}+\sup _{\chi \in S_{h}} \frac{\left|\left(\nabla Q_{h} \psi, \nabla \pi_{h} \phi\right)\right|}{\|\chi\|}=I+I I .
\end{aligned}
$$

By the obvious error estimate for $\pi_{h}$ and Lemma 2.4 we have

$$
|I| \leq C h \sup _{\chi \in S_{h}} \frac{\left\|\nabla Q_{h} \psi\right\||\phi|_{2}}{\|\chi\|} \leq C h^{2}\|\psi\|
$$

To estimate $I I$, we first rewrite the numerator in the form (cf. (1.7)),

$$
\left(\nabla Q_{h} \psi, \nabla \pi_{h} \phi\right)=\epsilon_{h}\left(\psi, \pi_{h} \phi\right)=\left(\psi, \pi_{h} \phi\right)_{h}-\left(\psi, \pi_{h} \phi\right)=\sum_{\tau \in \mathcal{T}_{h}} \int_{\tau}\left(\pi_{h}(\psi \phi)-\psi \pi_{h} \phi\right) d x
$$

Denoting the vertices of $\tau$ by $z_{1}^{\tau}, z_{2}^{\tau}, z_{3}^{\tau}$ and setting $z_{4}^{\tau}=z_{1}^{\tau}, z_{0}^{\tau}=z_{3}^{\tau}$, and $u\left(z_{j}^{\tau}\right)=u_{j}$ (cf. Figure 1), we obtain after a simple calculation

$$
\int_{\tau}\left(\pi_{h}(\psi \phi)-\psi \pi_{h} \phi\right) d x=-\frac{|\tau|}{12} \sum_{j=1}^{3} \psi_{j}\left(\Delta^{\tau} \phi\right)_{j},
$$

where

$$
\left(\Delta^{\tau} \phi\right)_{j}=\phi_{j-1}-2 \phi_{j}+\phi_{j+1}, j=1,2,3 .
$$

Hence, if $\tau \in \Pi_{i}$, with $\left(\Delta^{\tau} \phi\right)\left(z_{i}\right)=\left(\Delta^{\tau} \phi\right)_{j}$ if $z_{j}^{\tau}=z_{i}$,

$$
\epsilon_{h}\left(\psi, \pi_{h} \phi\right)=-\sum_{z_{i} \in Z_{h}^{0}} \psi\left(z_{i}\right)\left(\Delta_{h}^{*} \phi\right)\left(z_{i}\right), \quad \text { where }\left(\Delta_{h}^{*} \phi\right)\left(z_{i}\right)=\frac{1}{12} \sum_{\tau \subset \Pi_{i}}|\tau|\left(\Delta^{\tau} \phi\right)\left(z_{i}\right) .
$$

We can look upon $\left(\Delta_{h}^{*} \phi\right)\left(z_{i}\right)$ as a finite difference approximation of $\Delta \phi$ at $z_{i}$, using the values of $\phi$ at the vertices of $\Pi_{i}$. Since $\left(\Delta_{h}^{*} \phi\right)\left(z_{i}\right)$ does not use information about 


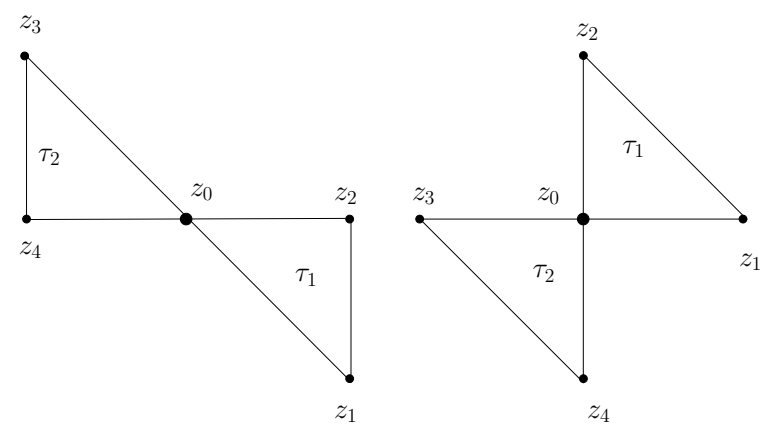

FIgURE 3. A pair of elements symmetric with respect to vertex $z_{0}$

the location of these vertices, it does not generally approximate the Laplacian $\Delta \phi$ at $z_{i}$. Such an example is the patch shown in Figure 1.

Now let $\mathcal{T}_{h}$ be symmetric at the vertex $z_{0}$, so that the patch $\Pi_{0}$ is symmetric around $z_{0}$. Then for the triangle $\tau_{1} \subset \Pi_{0}$ there is a triangle $\tau_{2} \subset \Pi_{0}$, symmetric to $\tau_{1}$ with respect to $z_{0}$ (see Figure 3), so that $\left|\tau_{1}\right|=\left|\tau_{2}\right|=|\tau|$. Also, for $\phi$ a linear function in $\Pi_{0}$, we have

$$
\left|\tau_{1}\right|\left(\Delta^{\tau_{1}} \phi\right)_{0}+\left|\tau_{2}\right|\left(\Delta^{\tau_{2}} \phi\right)_{0}=|\tau|\left(\phi_{1}+\phi_{2}-2 \phi_{0}+\phi_{3}+\phi_{4}-2 \phi_{0}\right)=0 .
$$

Thus, for a patch $\Pi_{i}$ which is symmetric with respect $z_{i}$ and $\phi$ linear in $\Pi_{i}$ we have $\left(\Delta_{h}^{*} \phi\right)_{i}=0$, since this expression will be a sum of symmetric pairs satisfying the relations (5.3). Applying the Bramble-Hilbert lemma we then obtain

$$
\left|\left(\Delta_{h}^{*} \phi\right)\left(z_{i}\right)\right| \leq C h^{2}\left|\Pi_{i}\right|^{1 / 2}\|\phi\|_{H^{2}\left(\Pi_{i}\right)} .
$$

Employing this estimate, for any $\psi \in S_{h}$, we get

$$
\begin{aligned}
& \left|\left(\nabla Q_{h} \psi, \nabla \pi_{h} \phi\right)\right|=\left|\epsilon_{h}\left(\psi, \pi_{h} \phi\right)\right| \leq\left|\sum_{z_{i} \in Z_{h}^{0}} \psi\left(z_{i}\right) \Delta_{h}^{*} \phi\left(z_{i}\right)\right| \\
& \leq C h^{2} \sum_{z_{i} \in Z_{h}^{0}}\left|\psi\left(z_{i}\right)\right|\left|\Pi_{i}\right|^{\frac{1}{2}}\|\phi\|_{H^{2}\left(\Pi_{i}\right)} \leq C h^{2}\|\psi\||\phi|_{2} \leq C h^{2}\|\psi\|\|\chi\|,
\end{aligned}
$$

and hence $|I I| \leq C h^{2}\|\psi\|$. Together with (5.2) this completes the proof.

\section{6. "Almost" Symmetric PARTitions in ONE DimEnSION}

In this section we shall consider the spatially one-dimensional analogue of the lumped mass method, and show that a nonsmooth data error estimate of type (4.2) holds for partitions which are somewhat more general than symmetric ones.

Let $\Omega=(0,1)$ and let $\mathcal{T}_{h}=\left\{I_{i}\right\}_{i=1}^{N}$, with $I_{i}=\left(x_{i-1}, x_{i}\right)$, be defined by the not necessarily uniform partition $0=x_{0}<x_{1}<\cdots<x_{N}=1$, and let $S_{h}$ be the set of the continuous piecewise linear functions over $\mathcal{T}_{h}$. We set $h_{i}=x_{i}-x_{i-1}$ and $h=\max _{i} h_{i}$. Using the quadrature formula,

$$
Q_{I_{i}, h_{i}}(f)=\frac{h_{i}}{2}\left(f\left(x_{i-1}\right)+f\left(x_{i}\right)\right) \approx \int_{x_{i-1}}^{x_{i}} f d x
$$


we now define the approximation of the inner product $(v, w)$ in $S_{h}$ by

$$
(\psi, \chi)_{h}=\sum_{i=1}^{N} Q_{I_{i}, h_{i}}(\psi \chi)=\sum_{i=1}^{N-1} \psi_{i} \chi_{i} \hbar_{i}, \quad \text { with } \hbar_{i}=\frac{1}{2}\left(h_{i}+h_{i+1}\right) .
$$

The lumped mass finite element method is then defined by

$$
\left(\bar{u}_{h, t}, \chi\right)_{h}+\left(\bar{u}_{h}^{\prime}, \chi^{\prime}\right)=0, \quad \forall \chi \in S_{h}, \quad \text { with } \bar{u}_{h}(0)=v_{h} .
$$

It is easy to demonstrate that the analogues of our results in Sections 35 remain valid also for (6.1). Here we will show that assumption (1.10) for the operator $Q_{h}$ holds for partitions which are "almost" symmetric in a sense to be defined below.

A direct computation shows that

$$
\begin{aligned}
\left(\left(Q_{h} \chi\right)^{\prime}, \psi^{\prime}\right) & =\epsilon_{h}(\chi, \psi)=(\chi, \psi)_{h}-(\chi, \psi) \\
& =-\frac{1}{6} \sum_{i=1}^{N-1}\left(h_{i+1}\left(\chi_{i+1}-\chi_{i}\right)-h_{i}\left(\chi_{i}-\chi_{i-1}\right)\right) \psi_{i}=\left(M_{h} \chi, \psi\right)_{h},
\end{aligned}
$$

where, taking into account that $\chi_{0}=\chi_{N}=0$, we have

$$
\left(M_{h} \chi\right)_{i}=-\frac{1}{6 \hbar_{i}}\left(h_{i+1}\left(\chi_{i+1}-\chi_{i}\right)-h_{i}\left(\chi_{i}-\chi_{i-1}\right)\right), \quad i=1, \ldots, N-1 .
$$

Similarly, direct computation of $\left(-\bar{\Delta}_{h} \chi, \psi\right)_{h}=\left(\chi^{\prime}, \psi^{\prime}\right)$ gives

$$
-\left(\bar{\Delta}_{h} \chi\right)_{i}=-\frac{1}{\hbar_{i}}\left(\frac{\chi_{i+1}-\chi_{i}}{h_{i+1}}-\frac{\chi_{i}-\chi_{i-1}}{h_{i}}\right), \quad i=1, \ldots, N-1,
$$

and we note that by the definition of the operator $-\bar{\Delta}_{h}$ we have

$$
\left(\left(Q_{h} \chi\right)^{\prime}, \psi^{\prime}\right)=-\left(\bar{\Delta}_{h} Q_{h} \chi, \psi\right)_{h}=\left(M_{h} \chi, \psi\right)_{h},
$$

so that

$$
-\bar{\Delta}_{h} Q_{h}=M_{h}, \quad \text { or } \quad Q_{h}=\left(-\bar{\Delta}_{h}\right)^{-1} M_{h} .
$$

Obviously, a partition that is symmetric with respect to each of its nodes is uniform, so that $h_{i}=h$ for all $i$. In this case (6.2) and (6.3) imply $6 h^{-2} M_{h}=-\bar{\Delta}_{h}$ and $Q_{h}=\frac{1}{6} h^{2} I$, where $I$ is the identity operator, and hence assumption (1.10) is satisfied. More generally, we have the following lemma which easily follows from (6.2) and (6.3) by checking the coefficients. Here, for $\widetilde{\omega}=\left(\omega_{1}, \ldots, \omega_{N-1}\right)$ and $\chi \in S_{h}$, we define $\widetilde{\omega} \chi \in S_{h}$ by $(\widetilde{\omega} \chi)_{i}=\omega_{i} \chi_{i}$. Further, we set $\left(\widetilde{h}^{2}\right)_{i}=h_{i}^{2}, i=$ $1, \ldots, N-1$.

Lemma 6.1. Let the operator $\bar{\partial}_{h}: S_{h} \rightarrow S_{h}$ be defined by $\left(\bar{\partial}_{h} \chi\right)_{i}=\left(\chi_{i}-\chi_{i-1}\right) / \hbar_{i}$, $i=1, \ldots, N-1$. Then

$$
M_{h} \chi=-\frac{1}{6} \bar{\Delta}_{h}\left(\widetilde{h}^{2} \chi\right)+\frac{1}{6} \bar{\partial}_{h}(\widetilde{\omega} \chi), \quad \text { where } \omega_{i}=h_{i+1}\left(1-\left(h_{i} / h_{i+1}\right)^{2}\right) .
$$

In the following theorem we shall consider families of partitions that are almost uniform in the sense that, uniformly in $h$,

$$
\left|\frac{h_{i}}{h_{i+1}}-1\right| \leq C h, \quad i=1, \ldots, N-1 .
$$

Theorem 6.1. If (6.5) holds, we have for $Q_{h}=\left(-\bar{\Delta}_{h}\right)^{-1} M_{h}$, uniformly in $h$,

$$
\left\|Q_{h} \chi\right\| \leq C h^{2}\|\chi\|, \quad \text { for } \chi \in S_{h} .
$$


Proof. By (6.4) and Lemma 6.1] we have

$$
\left(Q_{h} \chi\right)_{i}=\left(\left(-\bar{\Delta}_{h}\right)^{-1} M_{h} \chi\right)_{i}=\frac{1}{6} h_{i}^{2} \chi_{i}+\frac{1}{6}\left(\left(-\bar{\Delta}_{h}\right)^{-1} \bar{\partial}_{h}(\widetilde{\omega} \chi)\right)_{i}=I_{i}+I I_{i} .
$$

Clearly, $\|I\|_{h} \leq \frac{1}{6} h^{2}\|\chi\|_{h}$. To deal with $I I$, we note that $\left(-\bar{\Delta}_{h}\right)^{-1}$, and hence also $\left(-\bar{\Delta}_{h}\right)^{-1 / 2}$, is bounded in $\|\cdot\|_{h}$, uniformly in $h$, and we shall show the following:

Lemma 6.2. With our definitions above we have, uniformly in $h$,

$$
\left\|\left(-\bar{\Delta}_{h}\right)^{-1 / 2} \bar{\partial}_{h} \chi\right\|_{h} \leq C\|\chi\|_{h}, \quad \text { for } \chi \in S_{h} .
$$

Using this lemma we find, since $\left|\omega_{i}\right| \leq C h^{2}$, that $\|I I\|_{h} \leq C\|\widetilde{\omega} \chi\|_{h} \leq C h^{2}\|\chi\|_{h}$, which completes the proof of the Theorem 6.1.

Proof of Lemma 6.2. . We have

$$
\left\|\left(-\bar{\Delta}_{h}\right)^{-1 / 2} \bar{\partial}_{h} \chi\right\|_{h}=\sup _{\psi \in S_{h}} \frac{\left(\left(-\bar{\Delta}_{h}\right)^{-1 / 2} \bar{\partial}_{h} \chi, \psi\right)_{h}}{\|\psi\|_{h}}=\sup _{\psi \in S_{h}} \frac{\left(\bar{\partial}_{h} \chi,\left(-\bar{\Delta}_{h}\right)^{-1 / 2} \psi\right)_{h}}{\|\psi\|_{h}} .
$$

Consider for $\phi \in S_{h}$, with $\partial_{h} \chi_{i}=\left(\chi_{i+1}-\chi_{i}\right) / \hbar_{i}$,

$\left(\bar{\partial}_{h} \chi, \phi\right)_{h}=\sum_{i=1}^{N}\left(\chi_{i}-\chi_{i-1}\right) \phi_{i}=-\sum_{i=0}^{N-1} \chi_{i}\left(\phi_{i+1}-\phi_{i}\right)=-\left(\chi, \partial_{h} \phi\right)_{h} \leq\|\chi\|_{h}\left\|\partial_{h} \phi\right\|_{h}$.

Note that

$$
\left\|\partial_{h} \phi\right\|_{h}^{2} \leq C\left\|\phi^{\prime}\right\|^{2}=C\left(-\bar{\Delta}_{h} \phi, \phi\right)_{h}, \quad \text { with } C>0 .
$$

Now choose $\phi=\left(-\bar{\Delta}_{h}\right)^{-1 / 2} \psi$ to find

$$
\left\|\partial_{h}\left(-\bar{\Delta}_{h}\right)^{1 / 2} \psi\right\|_{h}^{2} \leq C\left(\left(-\bar{\Delta}_{h}\right)\left(-\bar{\Delta}_{h}\right)^{-1 / 2} \psi,\left(-\bar{\Delta}_{h}\right)^{-1 / 2} \psi\right)_{h}=C\|\psi\|_{h}^{2} .
$$

Hence

$$
\left(\bar{\partial}_{h} \chi,\left(-\bar{\Delta}_{h}\right)^{-1 / 2} \psi\right)_{h} \leq C\|\chi\|_{h}\|\psi\|_{h}
$$

which completes the proof.

As in Theorem 4.2 the result of Theorem 6.1 implies a nonsmooth data error estimate of the form (4.2) for $v_{h}=P_{h} v$.

\section{Counterexamples}

In this section we continue the discussion of the lumped mass method (6.1) in one space dimension and present two examples, where the necessary and sufficient condition for optimal convergence of Theorem 4.1 is not satisfied and hence the $O\left(h^{2}\right)$ nonsmooth data error estimate does not hold.

First, we consider a special nonuniform mesh by choosing $h=4 /(3 N)$, where $N$ is a positive integer divisible by 4 , and take

$$
h_{i}=\frac{1}{2} h, \quad \text { for } i \text { odd } \quad \text { and } \quad h_{i}=h, \quad \text { for } i \text { even, } \quad i=1, \ldots, N .
$$

Obviously, $\hbar_{i}=\frac{3}{4} h$. This mesh consists of $J=N / 2$ copies of the patch $\left(0, \frac{1}{2} h\right) \cup$ $\left(\frac{1}{2} h, \frac{3}{2} h\right)$ and is not symmetric with respect to any mesh-point; see Figure 4 .

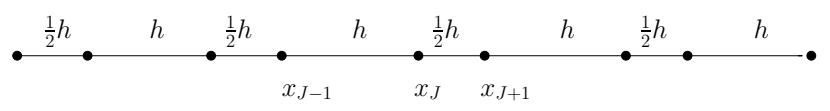

Figure 4. A nonsymmetric partition in one space dimension 
By Lemma 6.1 since $\omega_{i}=\frac{3}{4} h$, for $i$ odd, $\omega_{i}=-\frac{3}{2} h$, for $i$ even, we have

$$
M_{h} \chi=-\frac{1}{6} \bar{\Delta}_{h}\left(\widetilde{h}^{2} \chi\right)+\widetilde{M}_{h} \chi
$$

where

$$
\left(\widetilde{M}_{h} \chi\right)_{i}=\frac{1}{6} \begin{cases}\chi_{i}+2 \chi_{i-1}, & \text { for } i \text { odd, } \\ -2 \chi_{i}-\chi_{i-1}, & \text { for } i \text { even. }\end{cases}
$$

In view of Theorem 4.1 the following proposition will show that the $O(h)$ error estimate for $t>0$ in Theorem 4.3 is best possible.

Proposition 7.1. Let $\mathcal{T}_{h}$ be defined by (7.1). For the lumped mass initial value problem (6.1) with $v_{h}=\sum_{j=0}^{\frac{1}{2} J-1}\left(\Phi_{J+2 j}-2 \Phi_{J+2 j+1}\right)$, where $\Phi_{j}$ is the nodal basis function at $x_{j}$, we have, for each $t>0$ and $h$ small,

$$
\left\|\bar{E}_{h}(t) \bar{\Delta}_{h} Q_{h} v_{h}\right\|_{h} \geq c h\left\|v_{h}\right\|_{h}, \quad \text { with } c=c(t)>0 \text {. }
$$

Proof. In view of (7.2) and (6.4) we have

$$
Q_{h} v_{h}=\left(-\bar{\Delta}_{h}\right)^{-1} M_{h} v_{h}=-\frac{1}{6} \widetilde{h}^{2} v_{h}+\left(-\bar{\Delta}_{h}\right)^{-1} \widetilde{M}_{h} v_{h}
$$

Since, by $\bar{E}_{h}(t) \bar{\Delta}_{h}=D_{t} \bar{E}_{h}(t)$ and Lemma 2.2 ,

$$
t\left\|\bar{E}_{h}(t) \bar{\Delta}_{h}\left(\widetilde{h}^{2} v_{h}\right)\right\| \leq C\left\|\widetilde{h}^{2} v_{h}\right\| \leq C h^{2}\left\|v_{h}\right\|, \quad \text { for } t>0,
$$

it will suffice to consider the last term on the right of (7.4). We find at once from (7.3) that $\widetilde{M}_{h}\left(\Phi_{2 j}-2 \Phi_{2 j+1}\right)=\left(\Phi_{2 j+2}-\Phi_{2 j}\right) / 3$, where we have set $\Phi_{N} \equiv 0$, so that

$$
\widetilde{M}_{h} v_{h}=\sum_{j=0}^{\frac{1}{2} J-1} \widetilde{M}_{h}\left(\Phi_{J+2 j}-2 \Phi_{J+2 j+1}\right)=\frac{1}{3} \sum_{j=0}^{\frac{1}{2} J-1}\left(\Phi_{J+2 j+2}-\Phi_{J+2 j}\right)=-\frac{1}{3} \Phi_{J} .
$$

Hence, with $\bar{\lambda}_{j}^{h}$ and $\bar{\phi}_{j}^{h}$ being the eigenfunction and eigenvalues of $-\bar{\Delta}_{h}$, since $x_{J}=\frac{1}{2}$ and hence $\left(\widetilde{M}_{h} v_{h}, \bar{\phi}_{1}^{h}\right)_{h}=\frac{1}{3}\left(\Phi_{J}, \bar{\phi}_{1}^{h}\right)_{h}=\frac{1}{4} h \bar{\phi}_{1}^{h}\left(\frac{1}{2}\right)$,

$$
\left\|\bar{E}_{h}(t) \bar{\Delta}_{h}\left(\left(-\bar{\Delta}_{h}\right)^{-1} \widetilde{M}_{h} v_{h}\right)\right\|_{h}^{2}=\sum_{j=1}^{N-1} e^{-2 \bar{\lambda}_{j}^{h} t}\left(\widetilde{M}_{h} v_{h}, \bar{\phi}_{j}^{h}\right)_{h}^{2} \geq \frac{1}{16} h^{2} e^{-2 \bar{\lambda}_{1}^{h} t} \bar{\phi}_{1}^{h}\left(\frac{1}{2}\right)^{2} .
$$

Since, as is easily seen, $\left\|v_{h}\right\|_{h}=\frac{\sqrt{5}}{2}$, the proof is completed by showing that the last expression is bounded below by $c(t) h^{2}$.

Let $\phi_{1}(x)=\sqrt{2} \sin \pi x$ be the eigenfunction of $-u^{\prime \prime}=\lambda u$, corresponding to the first eigenvalue $\lambda_{1}=\pi^{2}$. We shall need the fact that

$$
\left\|\bar{\phi}_{1}^{h}-\phi_{1}\right\|_{H^{1}}=O(h) \quad \text { and } \quad \bar{\lambda}_{1}^{h} \rightarrow \lambda_{1} \text {, as } h \rightarrow 0
$$

see e.g. 4, pp. 87-92]. Using this, we have

$$
\bar{\phi}_{1}^{h}\left(\frac{1}{2}\right) \geq \phi_{1}\left(\frac{1}{2}\right)-\left\|\bar{\phi}_{1}^{h}-\phi_{1}\right\|_{L_{\infty}} \geq \sqrt{2}-\left|\bar{\phi}_{h}^{1}-\phi_{1}\right|_{H^{1}} \geq \sqrt{2}-C h,
$$

which shows our claim. The proof is now complete.

Next we give a second example of a partition $\mathcal{T}_{h}$ for which the optimal order error estimate (4.2) does not hold, although $\mathcal{T}_{h}$ is symmetric with respect to all nodes of $\mathcal{T}_{h}$ but one. Let $J / N=3 / 5$ and $h=3 /(4 J)$, and let $\mathcal{T}_{h}$ be defined by (see Figure 50

$$
h_{j}=x_{j}-x_{j-1}=h, \quad \text { for } j \leq J \text { and } h_{j}=\frac{1}{2} h, \quad \text { for } J<j \leq N .
$$




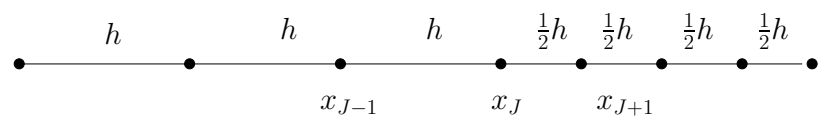

FIgURE 5. A nonsymmetric partition with respect to the point $x_{J}$

By Lemma 6.1 we may write, since $\omega_{J}=-\frac{3}{2} h$ and $\omega_{j}=0$ for $j \neq J$,

$$
M_{h} \chi=-\frac{1}{6} \bar{\Delta}_{h}\left(\widetilde{h}^{2} \chi\right)+\widetilde{M}_{h} \chi, \quad \text { where }\left(\widetilde{M}_{h} \chi\right)_{j}=\frac{h}{4 \hbar_{j}} \begin{cases}\chi_{J}, & \text { for } j=J+1, \\ -\chi_{J}, & \text { for } j=J, \\ 0, & \text { for } j \neq J, J+1,\end{cases}
$$

and it follows that

$$
\left(\widetilde{M}_{h} \chi, \psi\right)_{h}=\frac{1}{4} h \chi_{J}\left(\psi_{J+1}-\psi_{J}\right), \quad \text { for } \chi, \psi \in S_{h} .
$$

Proposition 7.2. Let $\mathcal{T}_{h}$ be defined by (7.6). For the lumped mass initial value problem (6.1), with $\bar{u}_{h}(0)=\Phi_{J}$, the nodal basis function at $x_{J}=3 / 4$, we have

$$
\left\|\bar{E}_{h}(t) \bar{\Delta}_{h} Q_{h} \Phi_{J}\right\|_{h} \geq c(t) h^{3 / 2}\left\|\Phi_{J}\right\|_{h}, \quad \text { with } c(t)>0, \quad \text { for } t>0, h \text { small. }
$$

Proof. The proof is similar to Theorem 7.1. Using (7.7) we get

$$
\left\|\bar{E}_{h}(t) \widetilde{M}_{h} \Phi_{J}\right\|_{h}^{2} \geq e^{-2 t \bar{\lambda}_{1}^{h}}\left(\widetilde{M}_{h} \Phi_{J}, \bar{\phi}_{1}^{h}\right)_{h}^{2} \geq \frac{1}{16} e^{-2 \bar{\lambda}_{1}^{h} t} h^{2}\left(\bar{\phi}_{1, J+1}^{h}-\bar{\phi}_{1, J}^{h}\right)^{2} .
$$

Since (7.5) implies $\left|\left(\bar{\phi}_{1, J+1}^{h}-\bar{\phi}_{1, J}^{h}\right)-\left(\phi_{1, J+1}-\phi_{1, J}\right)\right| \leq C h^{3 / 2}$ and for $\phi_{1}=$ $\sqrt{2} \sin (\pi x)$ we have $\left|\phi_{1, J+1}-\phi_{1, J}\right| \geq \frac{\sqrt{2}}{2} h \pi\left|\cos \left(\pi x_{J}\right)\right|=\frac{1}{2} h \pi$, it follows that

$$
\left\|\bar{E}_{h}(t) \widetilde{M}_{h} \Phi_{J}\right\|_{h} \geq c(t) h^{2}=c(t) h^{3 / 2}\left\|\Phi_{J}\right\|_{h},
$$

since $\left\|\Phi_{J}\right\|_{h}=(3 h / 4)^{1 / 2}$. The proof is now complete.

\section{Some Fully Discrete SCHEMES}

In this final section we discuss briefly the generalization of our above results for the spatially semidiscrete lumped mass method to some basic fully discrete schemes, namely the backward Euler and Crank-Nicolson methods.

With $k>0, t_{n}=n k, n=0,1, \ldots$, the backward Euler lumped mass method approximates $u\left(t_{n}\right)$ by $U^{n} \in S_{h}$ for $n \geq 0$ such that, with $\bar{\partial} U^{n}=\left(U^{n}-U^{n-1}\right) / k$,

$$
\left(\bar{\partial} U^{n}, \chi\right)_{h}+\left(\nabla U^{n}, \nabla \chi\right)=0, \quad \forall \chi \in S_{h}, \quad \text { for } n \geq 1, \quad \text { with } U^{0}=v_{h},
$$

or, with $A_{h}=-\bar{\Delta}_{h}$,

$$
\bar{\partial} U^{n}+A_{h} U^{n}=0, \quad \text { for } n \geq 1, \quad \text { with } U^{0}=v_{h} .
$$

Note that, for simplicity of notation, we write $U^{n}$ instead of the perhaps more natural $\bar{U}^{n}$, and similarly below, $E_{k h}$ instead of $\bar{E}_{k h}$.

We shall have use for the following abstract lemma, in the case $\mathcal{H}=S_{h}$, normed by $\|\cdot\|_{h}$, and $A=A_{h}$.

Lemma 8.1. Let $A$ be a linear, selfadjoint, positive definite operator in a Hilbert space $\mathcal{H}$, with compact inverse, let $\mathbf{u}=\mathbf{u}(t)$ be the solution of

$$
\mathbf{u}^{\prime}+A \mathbf{u}=0, \quad \text { for } t>0, \quad \text { with } \mathbf{u}(0)=\mathbf{v},
$$

and let $\mathbf{U}=\left\{\mathbf{U}^{n}\right\}_{n=0}^{\infty}$ be defined by

$$
\bar{\partial} \mathbf{U}^{n}+A \mathbf{U}^{n}=0, \quad \text { for } n \geq 1, \quad \text { with } \mathbf{U}^{0}=\mathbf{v} .
$$


Then, for $p=0,1,-1 \leq q \leq 3$, with $p+q \geq 0$, we have

$$
\left\|A^{p / 2}\left(\mathbf{U}^{n}-\mathbf{u}\left(t_{n}\right)\right)\right\| \leq C k t_{n}^{-(1-q / 2)}\left\|A^{(p+q) / 2} \mathbf{v}\right\|, \quad \text { for } n \geq 1 .
$$

Proof. Solving for $\mathbf{U}^{n}$ we may write (8.3) as $\mathbf{U}^{n}=(I+k A)^{-1} \mathbf{U}^{n-1}$ and hence

$$
\mathbf{U}^{n}=E_{k}^{n} \mathbf{v}, \quad \text { where } E_{k}=r(k A), \quad \text { with } r(\lambda)=1 /(1+\lambda) .
$$

Thus, since $\mathbf{u}\left(t_{n}\right)=e^{-t_{n} A} \mathbf{v}=e^{-n k A} \mathbf{v}$, we have

$$
A^{p / 2}\left(\mathbf{U}^{n}-\mathbf{u}\left(t_{n}\right)\right)=A^{p / 2} F_{n}(k A) \mathbf{v}, \quad \text { with } F_{n}(\lambda)=r^{n}(\lambda)-e^{-n \lambda},
$$

and therefore, by eigenfunction expansion and Parseval's relation,

$$
\begin{aligned}
\left\|A^{p / 2}\left(\mathbf{U}^{n}-\mathbf{u}\left(t_{n}\right)\right)\right\| & \leq \sup _{\lambda \in \sigma(A)}\left|\lambda^{-q / 2} F_{n}(k \lambda)\right|\left\|A^{(p+q) / 2} \mathbf{v}\right\| \\
& =k^{q / 2} \sup _{\lambda \in \sigma(k A)}\left|\lambda^{-q / 2} F_{n}(\lambda)\right|\left\|A^{(p+q) / 2} \mathbf{v}\right\| .
\end{aligned}
$$

Hence, since $k^{q / 2} n^{-(1-q / 2)}=k t_{n}^{-(1-q / 2)}$, it suffices for the proof of (8.4) to show

$$
\lambda^{-q / 2}\left|F_{n}(\lambda)\right| \leq C n^{-(1-q / 2)}, \quad \text { for } \lambda>0, n \geq 1 .
$$

For $0<\lambda \leq 1$ we have $|r(\lambda)| \leq e^{-c \lambda}$, with $c>0$, and $\left|r(\lambda)-e^{-\lambda}\right| \leq C \lambda^{2}$. Hence

$$
\begin{aligned}
\lambda^{-q / 2}\left|F_{n}(\lambda)\right| & \leq \lambda^{-q / 2}\left|r(\lambda)-e^{-\lambda}\right|\left|\sum_{j=0}^{n-1} r^{n-1-j}(\lambda) e^{-j \lambda}\right| \\
& \leq C \lambda^{2-q / 2} n e^{-c n \lambda} \leq C n^{-(1-q / 2)}, \quad \text { for } n \geq 1 .
\end{aligned}
$$

For $\lambda \geq 1$ we have $|r(\lambda)| \leq e^{-c}$, with $c>0$, and since $\lambda^{-q / 2}|r(\lambda)| \leq C$, we find

$$
\lambda^{-q / 2}\left|F_{n}(\lambda)\right| \leq \lambda^{-q / 2}|r(\lambda)||r(\lambda)|^{n-1}+\lambda^{-q / 2} e^{-n \lambda} \leq C n^{-(1-q / 2)}, \quad \text { for } n \geq 1,
$$

which shows (8.5) and thus completes the proof.

We now show some optimal order error estimates for (8.1) with initial data in $\dot{H}^{2}$ and $\dot{H}^{1}$, and for initial data only in $L_{2}$, if (1.10) holds for $Q_{h}$.

Theorem 8.1. Let $U$ be the solution of (8.1), and $u$ that of (1.1). Then

(8.6) $\left\|U^{n}-u\left(t_{n}\right)\right\| \leq C\left(h^{2}+k\right) t_{n}^{-1+q / 2}|v|_{q}$, for $n>0, q=1,2$, if $v_{h}=R_{h} v$.

Further, if (1.10) holds for $Q_{h}$,

$$
\left\|U^{n}-u\left(t_{n}\right)\right\| \leq C\left(h^{2}+k\right) t_{n}^{-1}\|v\|, \text { for } n>0, \quad \text { if } v_{h}=P_{h} v .
$$

Proof. We start with the estimates (8.6) and split the error as

$$
U^{n}-u\left(t_{n}\right)=\left(U^{n}-\bar{u}_{h}\left(t_{n}\right)\right)+\left(\bar{u}_{h}\left(t_{n}\right)-u\left(t_{n}\right)\right)=\beta_{n}+\eta_{n} .
$$

In view of Theorem 3.1 $\eta_{n}$ is bounded as required. We obtain, by Lemma 8.1

$$
\left\|\beta_{n}\right\|_{h}=\left\|U^{n}-\bar{u}_{n}\left(t_{n}\right)\right\|_{h} \leq C k t_{n}^{-(1-q / 2)}\left\|A_{h}^{q / 2} R_{h} v\right\|_{h} \leq C k t^{-(1-q / 2)}|v|_{q}, q=1,2,
$$

where the last inequality follows from $\left\|A_{h}^{1 / 2} R_{h} v\right\|_{h}=\left\|\nabla R_{h} v\right\| \leq|v|_{1}$ and

$$
\left\|A_{h} R_{h} v\right\|_{h}^{2}=\left(\nabla R_{h} v, \nabla A_{h} R_{h} v\right)=\left(\nabla v, \nabla A_{h} R_{h} v\right)=-\left(\Delta v, A_{h} R_{h} v\right),
$$

for $q=1,2$, respectively. This completes the proof of (8.6).

We turn now to (8.7). Estimating $\eta_{n}$ by Theorem 4.2, it remains to bound $\beta_{n}$ as stated. Employing Lemma 8.1, we have

$$
\left\|\beta_{n}\right\|_{h}=\left\|U^{n}-\bar{u}_{h}\left(t_{n}\right)\right\|_{h} \leq C k t_{n}^{-1}\left\|P_{h} v\right\|_{h} \leq C k t_{n}^{-1}\|v\| .
$$


For the gradient of the error we have the following smooth and nonsmooth data error estimates, without additional assumptions on the triangulations.

Theorem 8.2. Let $U$ be the solution of (8.1), and $u$ that of (1.1). Then

$$
\left\|\nabla\left(U^{n}-u\left(t_{n}\right)\right)\right\| \leq \begin{cases}C(h+k)|v|_{3}, & \text { if } v_{h}=R_{h} v \\ C\left(h t_{n}^{-1}+k t_{n}^{-3 / 2}\right)\|v\|, & \text { if } v_{h}=P_{h} v .\end{cases}
$$

Proof. The estimates needed for $\eta_{n}$ are contained in Theorems 3.1 and 4.4. To bound $\beta_{n}$ in the smooth data case, we first show the error bound for $v_{h}=\widetilde{v}_{h}=$ $-A_{h}^{-1} R_{h} \Delta v$. Since $\|\nabla \chi\|=\left\|A_{h}^{1 / 2} \chi\right\|_{h}$ for $\chi \in S_{h}$, we then have, by Lemma 8.1 .

$$
\left\|\nabla \beta_{n}\right\| \leq C k\left\|A_{h}^{3 / 2} \widetilde{v}_{h}\right\|_{h} \leq C k\left\|A_{h}^{1 / 2} R_{h} \Delta v\right\|_{h}=C k\left\|\nabla\left(R_{h} \Delta v\right)\right\| \leq C k|v|_{3} .
$$

In order to complete the proof it suffices to show

$$
\left\|\nabla\left(R_{h} v-\widetilde{v}_{h}\right)\right\| \leq C h|v|_{3} .
$$

In fact, setting $E_{k h}=\left(I+k A_{h}\right)^{-1}$ we have

$$
\left\|\nabla E_{k h}^{n} \chi\right\|=\left\|A_{h}^{1 / 2} E_{k h}^{n} \chi\right\|_{h} \leq\left\|A_{h}^{1 / 2} \chi\right\|_{h}=\|\nabla \chi\|,
$$

and hence

$$
\left\|\nabla E_{k h}^{n}\left(v_{h}-\widetilde{v}_{h}\right)\right\| \leq\left\|\nabla\left(v_{h}-v\right)\right\|+\left\|\nabla\left(v-R_{h} v\right)\right\|+\left\|\nabla\left(R_{h} v-\widetilde{v}_{h}\right)\right\| \leq C h|v|_{3} .
$$

The estimate (8.10) follows from

$$
\begin{gathered}
\left(\nabla\left(\widetilde{v}_{h}-R_{h} v\right), \nabla \chi\right)=-\left(R_{h} \Delta v, \chi\right)_{h}+(\Delta v, \chi)=-\epsilon_{h}\left(R_{h} \Delta v, \chi\right)-\left(\left(R_{h}-I\right) \Delta v, \chi\right) \\
\leq C h\left\|R_{h} \Delta v\right\|\|\nabla \chi\|+C h\|\nabla \Delta v\|\|\chi\| \leq C h|v|_{3}\|\nabla \chi\|, \quad \text { for } \chi \in S_{h} .
\end{gathered}
$$

To bound $\beta_{n}$ for nonsmooth data, we use Lemma 8.1 with $p=1, q=-1$ to find

$$
\left\|\nabla \beta_{n}\right\| \leq C k t_{n}^{-3 / 2}\left\|P_{h} v\right\|_{h} \leq C k t_{n}^{-3 / 2}\|v\| .
$$

This completes the proof of the theorem.

We now turn to the Crank-Nicolson method, defined by

$$
\bar{\partial} U^{n}+A_{h} U^{n-\frac{1}{2}}=0, \text { for } n \geq 1, \text { with } U^{0}=v_{h}, \quad U^{n-\frac{1}{2}}=\frac{1}{2}\left(U^{n}+U^{n-1}\right) .
$$

This method does not have as advantageous smoothing properties as the backward Euler method, which is reflected in the following counterpart of Lemma 8.1.

Lemma 8.2. Let $A$ and $\mathbf{u}$ be as in Lemma 8.1 and let $\mathbf{U}=\left\{\mathbf{U}^{n}\right\}_{n=0}^{\infty}$ satisfy

$$
\bar{\partial} \mathbf{U}^{n}+A \mathbf{U}^{n-\frac{1}{2}}=0, \quad \text { for } n \geq 1, \quad \text { with } \mathbf{U}^{0}=\mathbf{v} .
$$

Then, for $p=0,1, q=1,2$, we have

$$
\left\|A^{p / 2}\left(\mathbf{U}^{n}-\mathbf{u}\left(t_{n}\right)\right)\right\| \leq C k^{2} t_{n}^{-(2-q)}\left\|A^{p / 2+q} \mathbf{v}\right\|, \quad \text { for } n \geq 1 .
$$

Proof. Here, as in the proof of Lemma 8.1 employing eigenvalue expansions, it suffices to show, for $F_{n}(\lambda)=r^{n}(\lambda)-e^{-n \lambda}$, with $r(\lambda)=\left(1-\frac{1}{2} \lambda\right) /\left(1+\frac{1}{2} \lambda\right)$, that

$$
\lambda^{-q}\left|F_{n}(\lambda)\right| \leq C n^{-(2-q)}, \quad \text { for } \lambda>0, n \geq 1, q=1,2 .
$$

For $0 \leq \lambda \leq 1$ we have $|r(\lambda)| \leq e^{-c \lambda}$, with $c>0$, and $\left|r(\lambda)-e^{-\lambda}\right| \leq C \lambda^{3}$, so that

$$
\lambda^{-q}\left|F_{n}(\lambda)\right| \leq C \lambda^{3-q} n e^{-c n \lambda} \leq C n^{-(2-q)}, \quad \text { for } 0<\lambda \leq 1, n \geq 1, q=1,2 .
$$

For $\lambda \geq 1$ we have $|r(\lambda)| \leq e^{-c / \lambda}$, with $c>0$, and hence

$$
\lambda^{-q}\left|F_{n}(\lambda)\right| \leq \lambda^{-q} e^{-c n / \lambda}+\lambda^{-q} e^{-n \lambda} \leq C n^{-(2-q)}, \quad \text { for } n \geq 1, q=1,2 \text {. }
$$


We now show optimal order error estimates, where this time we need to require $v \in \dot{H}^{4}$ for the error bound to hold uniformly down to $t=0$. Because of the limited smoothing in the Crank-Nicolson method, no error bound is given for $v$ only in $L_{2}$.

Theorem 8.3. Let $U$ be the solution of (8.11), and $u$ that of (1.1). Then, with $q=1,2$, we have

$$
\left\|U^{n}-u\left(t_{n}\right)\right\| \leq C\left(h^{2}+k^{2} t_{n}^{-(2-q)}\right)|v|_{2 q}, \quad \text { for } n \geq 1, \quad \text { if } \quad v_{h}=R_{h} v .
$$

Proof. With our new $U^{n}$ we may again split the error as in (8.8), and by Theorem $3.1 \eta_{n}$ is bounded as desired. To bound $\beta_{n}$, it suffices, using the stability of $E_{k h}^{n}=$ $r^{n}\left(k A_{h}\right)$, with $r\left(k A_{h}\right)=\left(1-\frac{1}{2} k A_{h}\right)\left(1+\frac{1}{2} k A_{h}\right)^{-1}$, and Lemma 8.2, to find $\widetilde{v}_{h}$ such that

$$
\left\|\widetilde{v}_{h}-R_{h} v\right\| \leq C h^{2}|v|_{2 q} \text { and }\left\|A_{h}^{q} \widetilde{v}_{h}\right\|_{h} \leq C|v|_{2 q} .
$$

For $q=2$ we may choose $\widetilde{v}_{h}=-A_{h}^{-1} R_{h} \Delta v$, because $\left\|A_{h}^{2} \widetilde{v}_{h}\right\|_{h} \leq C|v|_{4}$ and

$$
\left\|\widetilde{v}_{h}-R_{h} v\right\| \leq C\left\|\nabla\left(\widetilde{v}_{h}-R_{h} v\right)\right\| \leq C h^{2}|v|_{4},
$$

in which the latter inequality follows, using our definitions and Lemma 2.3. from

$$
\begin{gathered}
\left(\nabla\left(\widetilde{v}_{h}-R_{h} v\right), \nabla \chi\right)=-\left(R_{h} \Delta v, \chi\right)_{h}+(\Delta v, \chi)=-\epsilon_{h}\left(R_{h} \Delta v, \chi\right)-\left(\left(R_{h}-I\right) \Delta v, \chi\right) \\
\leq C h^{2}\left\|\nabla R_{h} \Delta v\right\|\|\nabla \chi\|+C h^{2}|\Delta v|_{2}\|\chi\| \leq C h^{2}|v|_{4}\|\nabla \chi\|, \quad \text { for } \chi \in S_{h} .
\end{gathered}
$$

For $q=1$, (8.13) is obviously satisfied with $\widetilde{v}_{h}=R_{h} v$, completing the proof.

We now show corresponding error bounds for the gradient of the error.

Theorem 8.4. Let $U$ be the solution of (8.11), and $u$ that of (1.1). Then, for $q=1,2$, we have

$$
\left\|\nabla\left(U^{n}-u\left(t_{n}\right)\right)\right\| \leq C\left(h+k^{2} t_{n}^{-(2-q)}\right)|v|_{2 q+1}, \text { for } n \geq 1, \text { if } v_{h}=R_{h} v
$$

Proof. Again, by Theorem 3.1, $\eta_{n}$ is bounded as desired. To estimate $\beta_{n}$, we now want to find $\widetilde{v}_{h}$ such that

$$
\left\|\nabla\left(\widetilde{v}_{h}-R_{h} v\right)\right\| \leq C h|v|_{2 q+1} \quad \text { and } \quad\left\|A_{h}^{1 / 2+q} \widetilde{v}_{h}\right\|_{h} \leq C|v|_{2 q+1} .
$$

For $q=2$ we choose $\widetilde{v}_{h}=A_{h}^{-2} R_{h} \Delta^{2} v$, and obtain

$$
\left\|A_{h}^{5 / 2} \widetilde{v}_{h}\right\|_{h}=\left\|A_{h}^{1 / 2} R_{h} \Delta^{2} v\right\|_{h}=\left\|\nabla\left(R_{h} \Delta^{2} v\right)\right\| \leq C|v|_{5}
$$

and the first part of (8.14) follows, with $A_{h} \psi=\chi$, from

$$
\begin{aligned}
\left(\nabla\left(\widetilde{v}_{h}-R_{h} v\right), \nabla \chi\right)= & \left(R_{h} \Delta^{2} v, \psi\right)_{h}-\left(\left(R_{h}-I\right) \Delta v, \chi\right)-\epsilon_{h}\left(R_{h} \Delta v, \chi\right)-\left(\Delta^{2} v, \psi\right) \\
= & \epsilon_{h}\left(R_{h} \Delta^{2} v, \psi\right)-\left(\left(R_{h}-I\right) \Delta v, \chi\right)-\epsilon_{h}\left(R_{h} \Delta v, \chi\right) \\
& +\left(\left(R_{h}-I\right) \Delta^{2} v, \psi\right) \leq C h|v|_{5}\|\nabla \chi\|, \quad \text { for } \chi \in S_{h} .
\end{aligned}
$$

For $q=1$ we take, as in the proof of Theorem $8.2, \widetilde{v}_{h}=-A_{h}^{-1} R_{h} \Delta v$, recalling from (8.9) and (8.10) that (8.14) then holds.

In order to produce optimal order convergence for initial data only in $L_{2}$, assuming $Q_{h}$ appropriate, one may modify the Crank-Nicolson scheme by taking the first two steps by the backward Euler method, which has a smoothing effect, to obtain the following result. The proof is analogous to those of Theorems 8.1 and 8.3 and uses the appropriate combination of Lemmas 8.1 and 8.2, cf. [7], Theorem 7.4. 
Theorem 8.5. Let $U^{n}$ be the defined by (8.1) for $n=1,2$, and by (8.11) for $n \geq 3$, and let $u$ be the solution of (1.1). Then, if $Q_{h}$ satisfies (1.10), we have

$$
\left\|U^{n}-u\left(t_{n}\right)\right\| \leq C\left(h^{2} t_{n}^{-1}+k^{2} t_{n}^{-2}\right)\|v\|, \quad \text { if } v_{h}=P_{h} v, \quad \text { for } n \geq 1 .
$$

We remark that if the mesh ratio condition $k \leq C h^{2}$ and the inverse assumption $\|\nabla \chi\| \leq C h^{-1}\|\chi\|$, for $\chi \in S_{h}$ hold, then the use of the two preliminary backward Euler steps above is not needed, and also, since $k^{2} t_{n}^{-2} \leq k t_{n}^{-1}$, the error bound may be written as $\left\|U^{n}-u\left(t_{n}\right)\right\| \leq C h^{2} t_{n}^{-1}\|v\|$. In fact, under these assumptions, the spectrum of $k A_{h}$ is bounded above and one easily shows that (8.12) holds also with $q=0$, which implies our claim. Similarly, if instead $k \leq C h^{5 / 3}$, then the spectrum

of $k A_{h}$ is bounded above by $C h^{-1 / 3}$, and one easily finds that one backward Euler step suffices to show $\left\|U^{n}-u\left(t_{n}\right)\right\| \leq C h^{2} t_{n}^{-3 / 2}\|v\|$.

\section{ACKNOWLEDGMENTS}

The research of R.D. Lazarov was supported in parts by US NSF Grants DMS0713829, DMS-1016525, the Pichoridis Distinguished Lectureship through the University of Crete in 2008, and by award KUS-C1-016-04, made by King Abdullah University of Science and Technology (KAUST).

\section{REFERENCES}

[1] P. Chatzipantelidis, R. D. Lazarov, and V. Thomée, Error estimates for a finite volume element method for parabolic equations in convex polygonal domains, Numerical Methods for Partial Differential Equations, 20(5), (2004), 650-674. MR2076342 (2005g:65122)

[2] P. Chatzipantelidis, R. D. Lazarov, V. Thomée, and L. B. Wahlbin, Parabolic finite element equations in nonconvex polygonal domains, BIT Numerical Mathematics, 46 (suppl. 5), (2006), 113-143. MR 2283311 (2007m:65084)

[3] C. M. Chen and V. Thomée, The lumped mass finite element method for a parabolic problem, J. Austral. Math. Soc. Ser. B, 26(3), (1985), 329-354. MR776320 (86m:65117)

[4] G. J. Fix, Effects of quadrature errors in finite element approximations of steady state, eigenvalue and parabolic problem, The Mathematical Foundations of the Finite Element Method with Applications to Partial Differential Equations, Ed. A. K. Aziz, Academic Press, New York, 1972, pp. 525-556. MR0413546 (54:1660)

[5] H. O. Kreiss, V. Thomée, and O. Widlund, Smoothing of initial data and rates of convergence for parabolic difference equations, Comm. Pure Appl. Math., 23(2), (1970), 241-252. MR.0251935 (40:5160)

[6] N. Saito, A holomorphic semigroup approach to the lumped mass finite element method, J. Comput. Appl. Math., 169(1), (2004), 71-85. MR2071261(2005e:65149)

[7] V. Thomée, Galerkin Finite Element Methods for Parabolic Problems, Springer-Verlag, Second Edition, Berlin, 2006. MR2249024 (2007b:65003)

Department of Mathematics, University of Crete, GR-71409 Heraklion, Greece

E-mail address: chatzipa@math.uoc.gr

Department of Mathematics, Texas A\&M University, College Station, TX 77843, USA, and Institute of Mathematics and Informatics, Bulgarian Academy of Sciences, Sofia, Bulgaria

E-mail address: lazarov@math.tamu.edu

Mathematical Sciences, Chalmers University of Technology and the University of Gothenburg, SE-412 96 Göteborg, Sweden, and Institute of Applied and Computational Mathematics, FORTH, Heraklion GR-71110, Greece

E-mail address: thomee@chalmers.se 